\title{
Architectural Considerations for a Minimum Mass, Minimum Energy, Artificial Gravity Environment
}

Theodore W. Hall

Chinese University of Hong Kong 



\title{
Architectural Considerations for a Minimum Mass, Minimum Energy, Artificial Gravity Environment
}

\author{
Theodore W. Hall \\ Chinese University of Hong Kong
}

Copyright $@ 2002$ Society of Automotive Engineers, Inc.

\begin{abstract}
Adaptation to artificial gravity is easiest when the radius and tangential velocity are large. To minimize mass and kinetic energy, economics pushes in the opposite direction, toward small radius and tangential velocity. To promote adaptation to such an environment, habitat designers must pay particular attention to the arrangement of internal activities, with respect to varying magnitudes and directions of centripetal and Coriolis accelerations. This involves the orientation of habitat modules with respect to the rotation axis, the modules' interior layout, and possibly other visual cues to help inhabitants maintain their own spatial orientation with respect to the spin.
\end{abstract}

\section{INTRODUCTION}

In planning for human excursions to Mars and beyond, there is considerable debate concerning the best strategy for health preservation, especially regarding countermeasures for the deconditioning that results from long exposure to micro gravity. Unlike current Earthorbital missions, where extended access to micro gravity is an objective, in interplanetary travel it's a nuisance. Although there may be some rationale to make the most of the situation by conducting micro-gravity work en route - "to turn lemons into lemonade" - the real goal is to get the people onto the planet in the best health with the least time spent in transit. Furthermore, activities most appropriate to the transit - such as measurements of the interplanetary environment - depend on neither micro gravity nor human presence.

Even in Earth orbit, human habitation of micro gravity is mostly for its own sake, to study the deconditioning and the efficacy of various countermeasures. The micro gravity environment per se offers little or no benefit to the crew, and it's debatable whether the immediate presence of the crew offers any benefit to other micro gravity research or development: tele-presence would be less disruptive. The main "benefit" of human habitation of micro gravity appears to be in entertainment and tourism, but these are short term activities. After a while, the glamour of confinement in micro gravity wears off [Oberg \& Oberg, 1986, p. 9, 129-130; Raymond, 1986, p. 2033].

Many proposals for human transit to Mars call for artificial gravity, to be induced by spinning some or all of the spacecraft [Capps, Fowler, \& Appleby, 1991; Schultz, Rupp, Hajos, \& Butler, 1989; Staehle, 1989; Welch, 1985; Zubrin, Baker, \& Gwynne, 1991]. This introduces many complications regarding structural loads and mass, launch and deployment, docking, propulsion, and configuration of communications dishes, solar collectors, and radiators. Engineers and mission planners balk at the complexity and cost of such designs and continue to seek alternatives, through some combination of pharmacology, exercise, intermittent use of an on-board short-arm centrifuge or other therapeutic devices, and post-flight rehabilitation.

Whatever strategy is chosen, the emphasis is likely to be on minimizing the cost while meeting the ethical demand to provide for the well-being of the crew. The cost of space travel is largely a function of the mass and energy invested in the transportation system. This includes the kinetic energy of the vessel as well as energy stores to effect any necessary velocity changes. For artificial gravity, this also includes the additional energy required to start and stop the rotation. Complexity also carries costs: in research, design, manufacturing, and testing. However, it's not clear that artificial gravity is necessarily more complex than the various suggested alternatives.

Therefore, in choosing for or against artificial gravity, it behooves designers to consider the most affordable configurations, that minimize mass and kinetic energy. A minimal artificial gravity environment is likely to require some adaptation on the part of the inhabitants. This is reasonable, considering that nearly half of all space travelers suffer some degree of "space sickness" while adapting to micro gravity [Connors, Harrison, \& Akins, 1985, p. 35-51; Merz, 1986, p. 2040-2041]. If artificial gravity is as difficult to adapt to, it still offers the advantage of health preservation and an easier return to natural gravity. 
MATHEMATICAL NOMENCLATURE - The following terms and symbols appear throughout this paper. Boldface indicates vectors; italics indicate scalar magnitudes; uppercase refers to the inertial frame, while lowercase refers to the rotating frame; measurement units are radians, meters, kilograms, and seconds, unless explicitly stated otherwise:

$\Omega$ : habitat's angular velocity.

$\mathbf{R}$ : habitat's radius of rotation.

$\mathbf{V}$ : habitat's tangential velocity $=\mathbf{\Omega} \times \mathbf{R}$

v : inhabitant's relative velocity.

$\mathbf{A}_{\text {cent }}$ : centripetal acceleration $=\boldsymbol{\Omega} \times(\boldsymbol{\Omega} \times \mathbf{R})=\mathbf{\Omega} \times \mathbf{V}$

$\mathbf{A}_{\text {Cor }}$ : Coriolis acceleration $=2 \boldsymbol{\Omega} \times \mathbf{v}$

$m:$ mass.

$E_{k}:$ kinetic energy $=m V^{2} / 2$

\section{COMFORT, MASS AND ENERGY}

Studies of human subjects in rotating environments [Clark \& Hardy, 1960; Cramer, 1985; Gilruth, 1969; Gordon \& Gervais, 1969; Graybiel, 1977; Hill \& Schnitzer, 1962; Stone, 1973] have identified several parameters pertaining to comfort, with the following limits:

- Minimum Radius. Because centripetal acceleration is proportional to radius, people will experience a head-tofoot gradient in the apparent gravity. There will also be a gradient in "vertical" (radial) motion, as when sitting, standing, or climbing between decks. The gradient per unit height is the inverse of the rotational radius. Thus, a maximum allowable gradient implies a minimum allowable radius.

- Minimum Tangential Velocity. This limit aims to reduce the gravitational distortion due to Coriolis acceleration. As a cross product of the environment's angular velocity, Coriolis acceleration is zero for motion parallel to the axis of rotation, and maximum for motion perpendicular to the axis. In the plane of rotation, the ratio of Coriolis to centripetal acceleration equals twice the ratio of a person's relative velocity to the environment's tangential velocity:

$$
\begin{aligned}
\frac{A_{\text {Cor }}}{A_{\text {cent }}} & =\frac{2 \Omega V}{\Omega V} \\
& =2 \frac{V}{V}
\end{aligned}
$$

To keep this ratio low, the environment's tangential velocity should be high.
- Minimum Centripetal Acceleration. This is the nominal "design gravity." The lower limit generally aims to provide adequate floor traction for mobility. The minimum required to preserve health remains unknown.

- Maximum Centripetal Acceleration. Exceeding $1 \mathrm{~g}$ is counter to comfort as well as economics.

- Maximum Angular Velocity. Rotating one's head about an axis that's not aligned with the environment's rotation involves a torque about a mutually perpendicular axis. This is similar to the torque required to reorient the axis of a spinning top or gyroscope. The torque acts on the vestibular organs of the inner ear, creating an illusion of rotation about that perpendicular axis. The illusion is approximately proportional in magnitude and direction to the cross product of the angular velocities of the environment and the head [Clark \& Hardy,1960, p. 109110; Lally, 1962, p. 57]. This mismatch of visual and vestibular senses of motion is a major cause of motion sickness [Connors et al., 1985, p. 35-51; Merz, 1986, p. 2040-2041]. The non-aligned rotations of the environment and the head are said to be "crosscoupled". To mitigate these effects, the angular velocity of the environment should be low.

As the formulas for $\mathbf{V}, \mathbf{A}_{\text {cent }}$ and $\mathbf{A}_{\text {cor }}$ show, all of these comfort limits relate, directly or indirectly, to the radius and tangential velocity of rotation. Moreover, they all favor a large radius and tangential velocity.

For a habitat with a given mass and centripetal acceleration, suspended from the axis of rotation by a structure of negligible mass, the rotational kinetic energy is directly proportional to the radius of rotation:

$$
\begin{aligned}
A_{\text {cent }} & =\Omega^{2} R \\
& =V^{2} / R \\
E_{k} & =m V^{2} / 2 \\
& =m A_{\text {cent }} R / 2
\end{aligned}
$$

If the suspension structure is not negligible, then its mass and kinetic energy also increase with radius. Thus, comfort in artificial gravity favors large mass and kinetic energy.

Economics pushes in the opposite direction, toward minimum energy, minimum mass, and small radius and tangential velocity. The artificial-gravity studies cited above have attempted to identify comfort zones that accommodate such conditions. Though they disagree on the limits, they inevitably encompass conditions in which the apparent gravity is distinctly different from Earth's. Comfort in this context denotes not luxury but merely adaptability and mitigation of symptoms of motion sickness. 
Table 1 summarizes, for various estimates of the comfort zone, the conditions that yield the minimum acceptable radius and tangential velocity. Some authors state these limits explicitly; for others, these derive from limits on minimum centripetal acceleration or maximum angular velocity. In each row, the two controlling parameters appear in boldface; the other two derive from them. Where authors have originally specified dimensions rounded to the nearest foot, Table 1 converts and rounds to the nearest 0.1 meter. As is customary, it lists centripetal acceleration in multiples of Earth gravity, and angular velocity in rotations per minute. The index numbers in the first column refer to the curves in Figure 1.

\section{THE GEOMETRY OF ARTIFICIAL GRAVITY}

FREE-FALL AND DEFLECTION - To illustrate the sort of gravity that results from these conditions, Figure 1 diagrams two simulations: dropping a particle from an initial height $\left(h_{0}\right)$ of 2 meters, and launching a particle
Table 1: Artificial gravity with minimum acceptable radius and tangential velocity. The controlling parameters appear in boldface; the others derive from them.

\begin{tabular}{|c|c|c|c|c|c|}
\hline \# & Author & $\begin{array}{c}\text { Min. } \\
R\end{array}$ & $\begin{array}{c}\text { Min. } \\
V \\
(\mathrm{~m} / \mathrm{s})\end{array}$ & $\begin{array}{l}\text { Min. } \\
\frac{A_{\text {cent }}}{9.81} \\
\text { (g) }\end{array}$ & $\begin{array}{l}\text { Max. } \\
\frac{30 \cdot \Omega}{\pi} \\
(\mathrm{rpm})\end{array}$ \\
\hline 1 & Hill \& Schnitzer [1962] & 14.6 & 6.1 & 0.26 & 4.0 \\
\hline 2 & Gilruth [1969] & 12.2 & 6.0 & 0.30 & 4.7 \\
\hline 3 & Gilruth "optimum" [1969] & 67.1 & 14.0 & 0.30 & 2.0 \\
\hline 4 & Gordon \& Gervais [1969] & 12.2 & 7.3 & 0.45 & 5.7 \\
\hline 5 & Stone [1973] & 15.2 & 10.2 & 0.69 & 6.4 \\
\hline 6 & Cramer [1985] & 23.3 & 7.3 & 0.23 & 3.0 \\
\hline
\end{tabular}

vertically from the floor with an initial velocity $\left(v_{0}\right)$ of 2 meters per second. On Earth, both particles would fall vertically. In a rotating habitat, viewed from an inertial reference, the particles "fall" on straight chords that cut

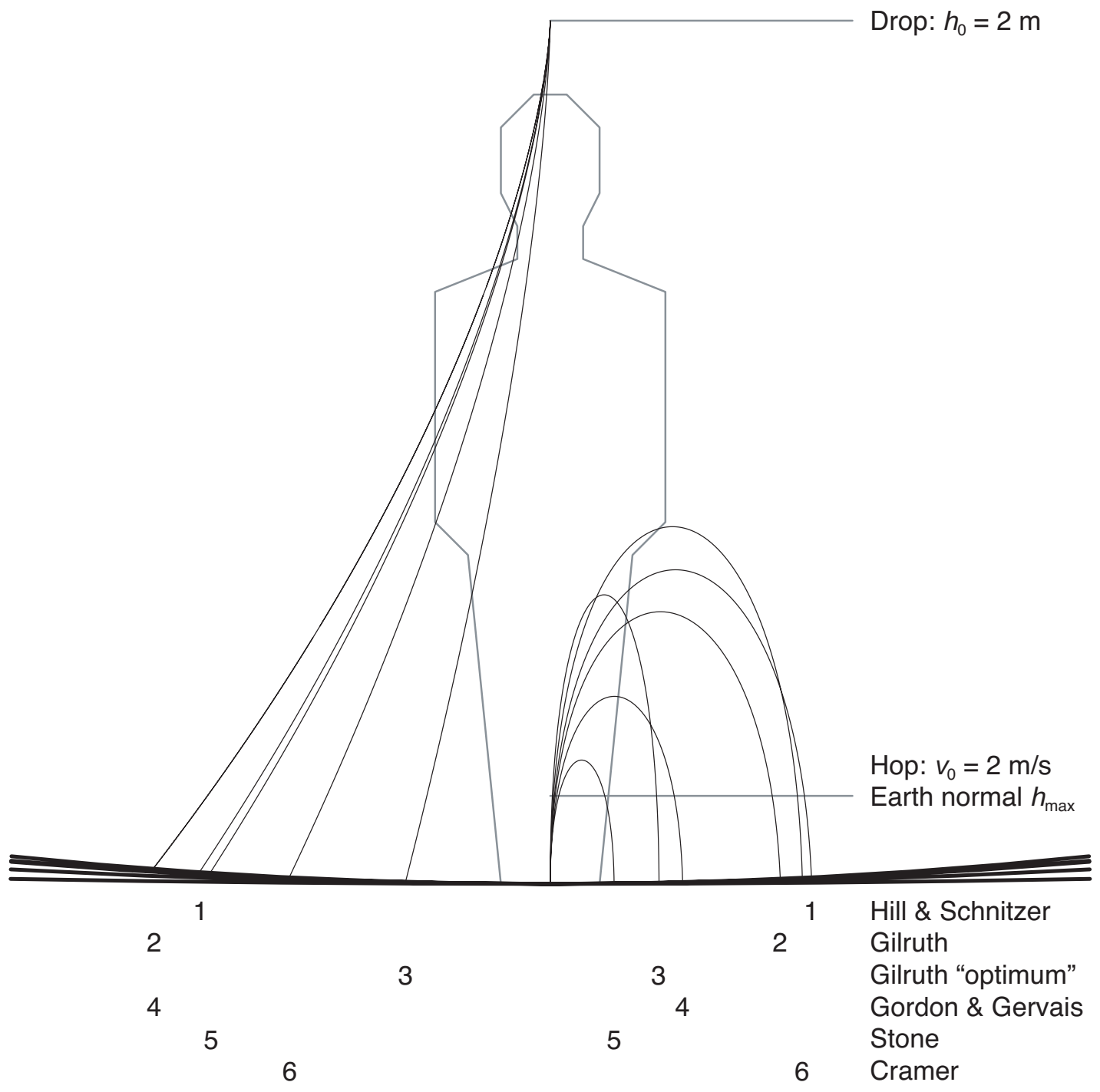

Figure 1: Relative motion of free-falling particles in the artificial-gravity environments specified in Table 1. Prograde is to the right; retrograde to the left. 
through a circle while the inhabitant rotates around the circumference. In the inhabitant's rotating frame of reference, the particles fall on curved paths that depend on the radius and tangential velocity of the habitat [Hall, 1999]:

- The retrograde deflection of a "dropping" particle $\left(v_{0}=0\right)$ depends on the ratio of its initial height to the floor radius, $h_{0} / R$. A larger radius yields a smaller ratio and a smaller deflection. The angular velocity and centripetal acceleration influence the particle's speed, but not its path. The path is an involute spiral (as if traced by the end-point of a thread unwinding from a spool of radius $R-h_{0}$ ).

- The prograde deflection of a "hopping" particle $\left(h_{0}=0\right)$ depends on the ratio of its initial relative velocity to the habitat's tangential velocity, $v_{0} / V$, and also on the floor radius. The velocity ratio determines the proportions of the path. A larger tangential velocity yields a smaller ratio and a narrower, more vertical path. The tangential velocity and radius together determine the centripetal acceleration and the absolute size of the path. A higher acceleration corresponds to stronger apparent gravity and a lower arc.

The "hop" and "drop" in Figure 1 represent motions within the sphere of typical human activity. The size $(h)$ and speed $(v)$ of humans are given, within a statistical range, by physiology. Selection and training may yield a crew with some variance from the general population, but these variables are essentially beyond the control of designers. The "normalcy" of the artificial gravity depends mostly on the designer's selection of radius and tangential velocity. For greatest comfort, these should be large relative to the size and speed of people. Nevertheless, Table 1 and Figure 1 indicate that considerable deviation from Earth-normal gravity is plausible.

\section{LINEAR MOTION AND APPARENT SLOPE - TO} minimize discomfort and promote human adaptation to such environments, habitat designers must pay particular attention to the arrangement of activities with respect to varying magnitudes and directions of centripetal and Coriolis accelerations. Figures 4 through 15 illustrate the gravitational distortions resulting from the Coriolis acceleration that accompanies linear motion in the plane of rotation.

For the sake of illustration, these figures assume a habitat similar in size and shape to TransHab [Kennedy, 1999], which was originally designed to serve as a microgravity "transit habitat" for a crew of six from Earth to Mars. Figure 2 provides a view of the TransHab design.

It is not the intention of this paper to advocate for or against the use of TransHab, either with or without artificial gravity. In fact, artificial gravity is specifically not

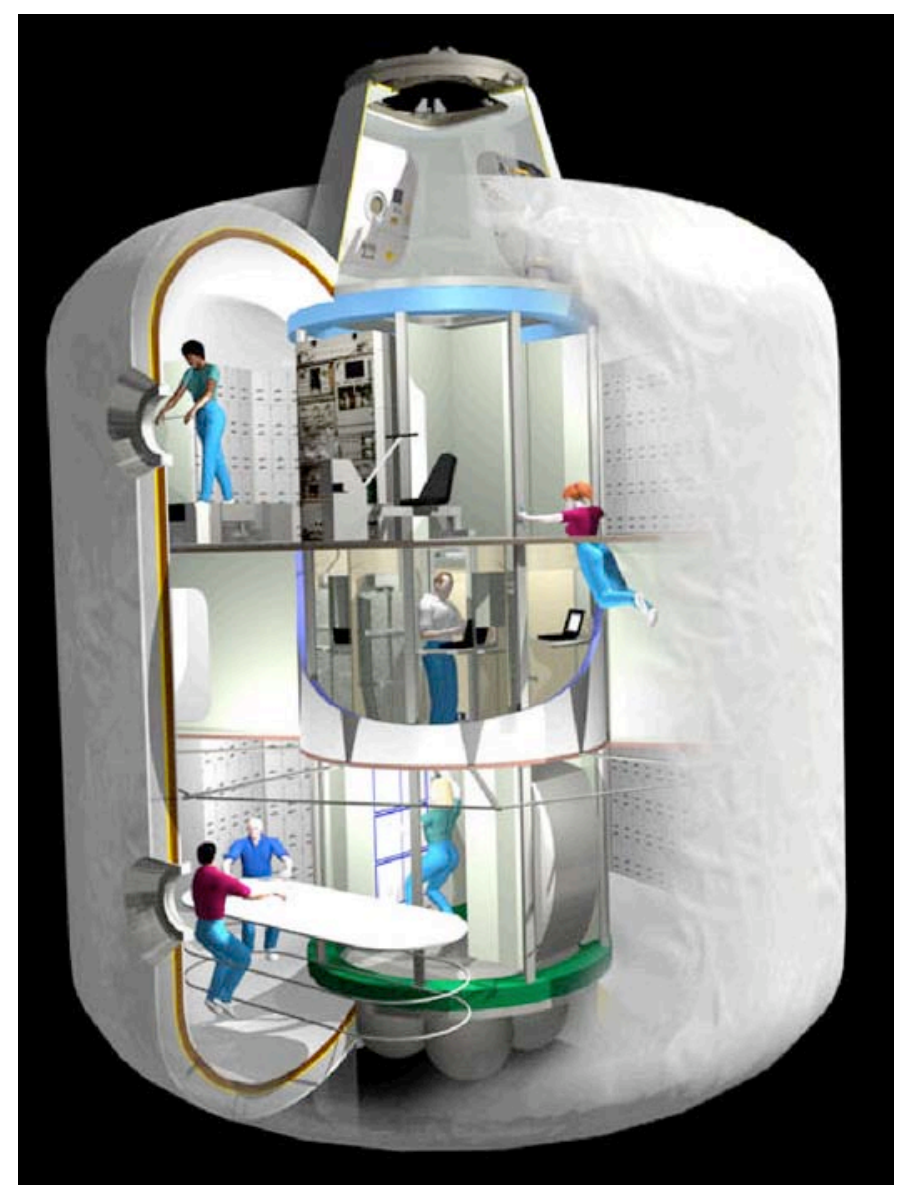

Figure 2: TransHab, NASA JSC S99-05363 [Kennedy 1999].

part of the TransHab program. Nevertheless, as a fairly recent and well-known design, it provides a plausible set of dimensions and a departure point for investigating artificial gravity in such environments. It's useful to consider what would happen if "something like TransHab" rotated to provide artificial gravity.

Accordingly, as a first guess, Figures 3 through 15 depict the habitat as a vertical cylinder with an inside diameter of 7.6 meters and a floor-to-floor height of 2.4 meters. This is not necessarily a minimum-mass environment. It does, however, conform to a serious proposal for an interplanetary habitat. Moreover, it's large enough to accommodate significant internal movement on all three axes. This allows one to study the effects of various relative motions in artificial gravity, which sheds some light on strategies for designing and orienting smaller habitats.

The overall spacecraft configuration is beyond the scope of this paper, which focuses specifically on the artificial gravitational environment within the habitat. Suffice to say that the habitat hangs below the rotation axis, supported by a tether, truss, or other structure that connects it to a counter mass. Figure 3 provides a schematic view of the rotation. 


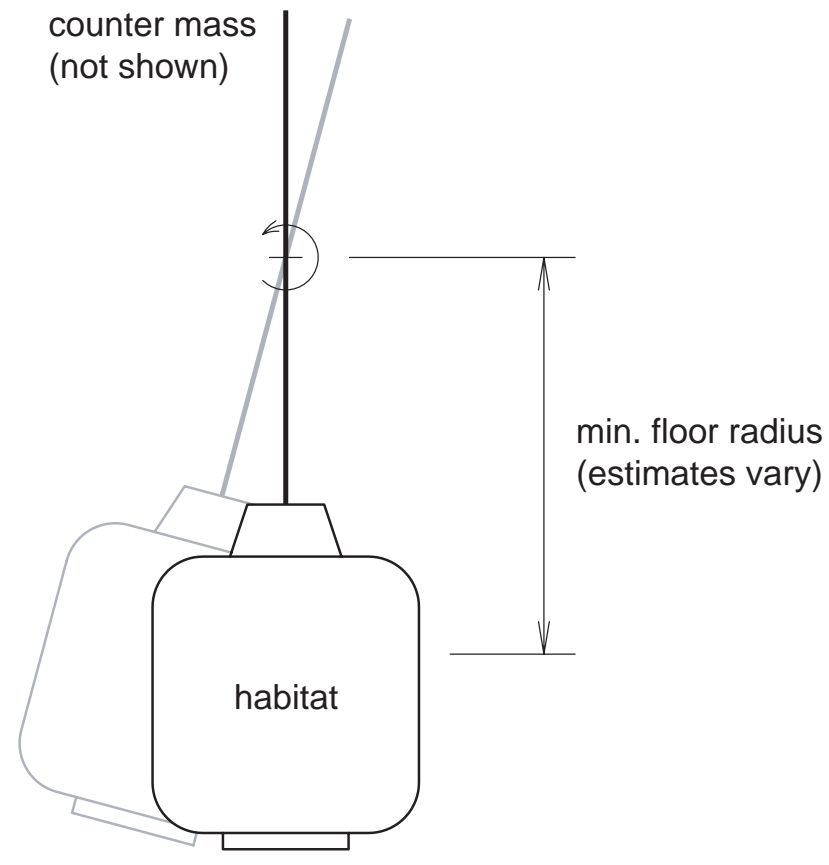

Figure 3: Rotating the habitat to induce artificial gravity.

Rather than investigate every condition listed in Table 1, Figures 4 through 15 focus on the "minimum of the minimums" and the "maximum of the minimums". Another look at the table reveals that Gilruth specifies both the most aggressive and the most conservative limits for minimizing radius and tangential velocity. Row 2 is acceptable only to Gilruth, whereas row 3 what Gilruth calls "optimum" - is acceptable to all of the cited authors. Accordingly, Figures 4 through 11 illustrate the gravity environment specified by row 2 , while Figures 12 through 15 illustrate row 3 for comparison. In each figure, the top floor of the module corresponds to the tabulated radius.

Measures of "slope angle" in the following paragraphs are rounded to the nearest $1^{\circ}$, calculated from the rotation parameters, the habitat dimensions, and assumed relative velocities of 1 meter per second for walking and 0.5 meter per second for climbing. The slope angles derive from the trigonometry of the acceleration components, as illustrated in the figures.

Figure 4 shows the centripetal, Coriolis, and total accelerations of an inhabitant walking prograde at a modest rate of 1 meter per second (about 2.2 miles per hour). The centripetal acceleration is always directed toward the center of rotation, with an angle and magnitude that varies with the inhabitant's position. The Coriolis acceleration is constant, perpendicular to the floor. The total acceleration converges on a point above the center of rotation, offset by the Coriolis component. The direction of the total acceleration defines the inhabitant's "up" vector. Its magnitude and slope vary with the inhabitant's distance from the center of the floor.
Figure 5 shows the situation from the inhabitant's frame of reference. Because the apparent floor slope (rise/run) is proportional to the path distance from the center of the floor, it follows the form of a catenary arch [Hall, 1999]. Under the conditions illustrated in Figures 4 and 5, the slope angle at the wall (3.8 meters from the center of the floor) is about $13^{\circ}$ (about $23 \%$ grade). To compare this with terrestrial architecture, the BOCA building code [BOCA, 1989, s. 817.6, 1306.3.2] allows a maximum slope angle of only about $7^{\circ}$ (1:8 slope, $12.5 \%$ grade) for means-of-egress ramps for healthy persons, and less than $5^{\circ}$ (1:12 slope, about $8.3 \%$ grade) for handicapped persons. The slope of the floor in this rotating module is clearly outside the range of comfortable terrestrial architecture.

Figure 6 shows the accelerations for the inhabitant after reversing direction, now walking retrograde at 1 meter per second. The Coriolis acceleration, as a vector product of the inhabitant's relative velocity, is also reversed. Thus, the total acceleration now converges on a point below the center of rotation. The apparent gravity (the magnitude of the acceleration) is less, but the apparent slope is greater.

Figure 7 shows the inhabitant's frame of reference. At its steepest, at the wall, the floor's apparent slope angle is about $25^{\circ}$ (nearly $47 \%$ grade).

Figures 8 and 9 show the inhabitant descending a vertical (radial) ladder at 0.5 meter per second from the top floor, while Figures 10 and 11 show him ascending at 0.5 meter per second from the bottom floor. Again, the Coriolis acceleration affects the apparent slope, as if the inhabitant were climbing a segment of a catenary arch. The apparent slope angle for descending is about $-81^{\circ}$; for ascending, about $83^{\circ}$. It's important to climb on top of the arch, rather than hang beneath it. As the figures show, one should descend on the prograde side of the ladder, and ascend on the retrograde side. This insures that the Coriolis acceleration presses the ladder against the climber, rather than pulling it away. In these figures, the opening in the floor is double wide to permit access to a single ladder from both sides. An alternative that would save floor area would be to provide two parallel ladders on opposite sides of a single, smaller opening. In any case, the plane of the ladder should be perpendicular to the plane of rotation.

Figures 12 through 15 repeat these four scenarios (prograde, retrograde, descending, and ascending motion) using a substantially larger rotational radius and tangential velocity: row 3 of Table 1 , instead of row 2 . Although the centripetal acceleration is the same in both cases $-0.3 \mathrm{~g}$ - the gravity is substantially more normal in the latter case. The floor slope angles are about $3^{\circ}$ (prograde) and $4^{\circ}$ (retrograde), while the ladder slope angles are about $\pm 86^{\circ}$ (ascending and descending). The floor slope, though not negligible, is at least within 


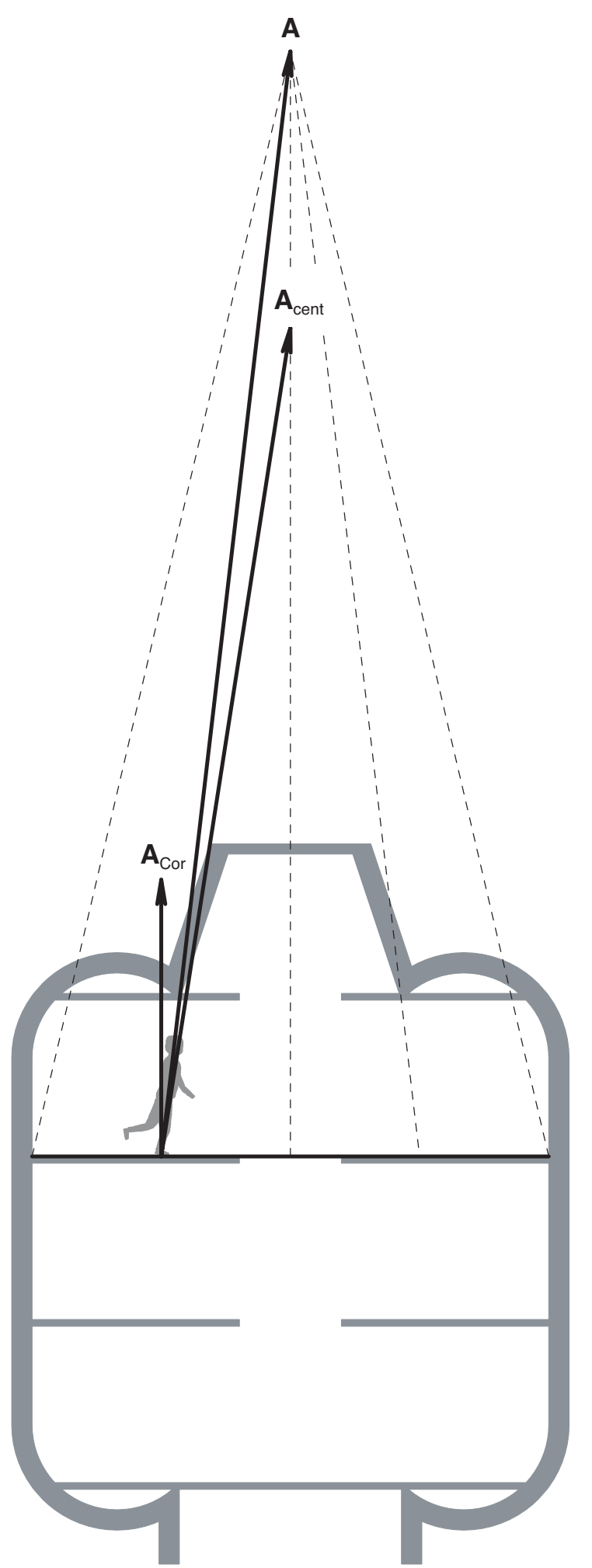

Figure 4: Centripetal, Coriolis, and total acceleration while walking prograde at $1 \mathrm{~m} / \mathrm{s}$, radius $12.2 \mathrm{~m}$, tangential velocity $6.0 \mathrm{~m} / \mathrm{s}$ (Table 1 row 2).

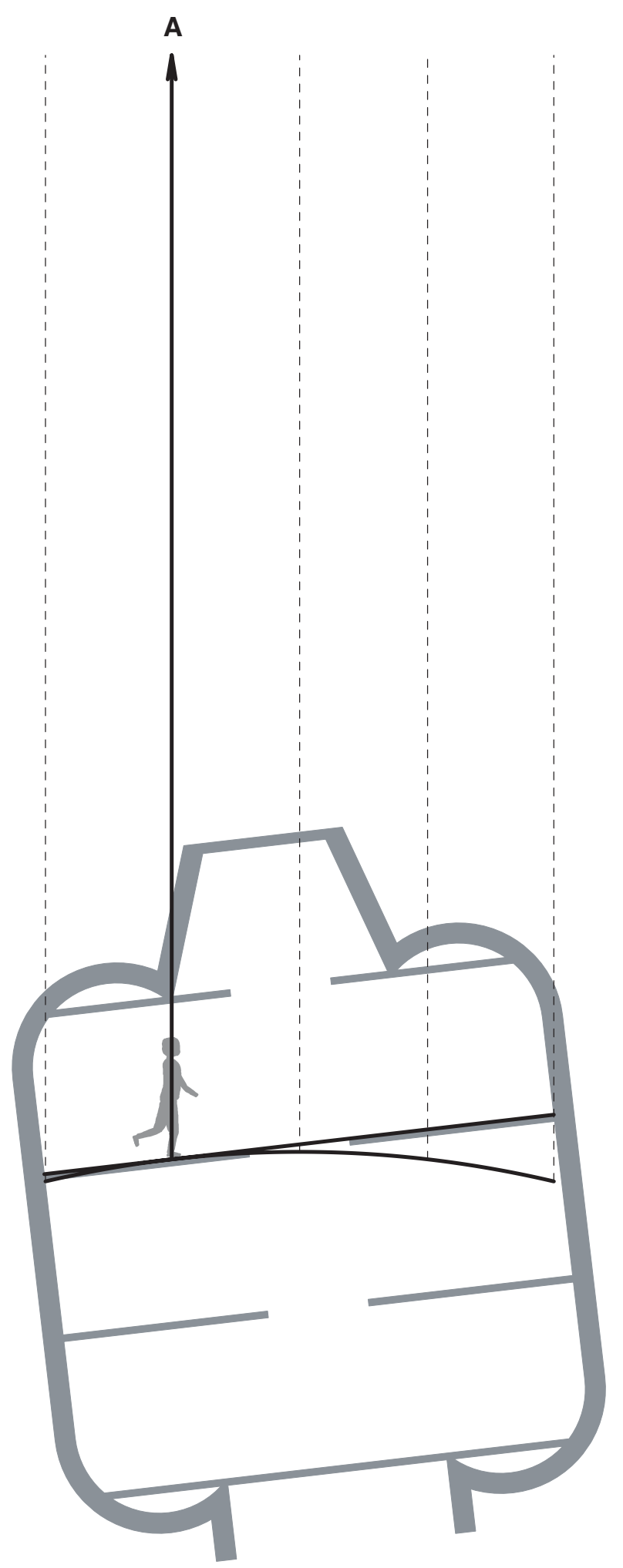

Figure 5: Apparent gravity for the inhabitant in Figure 4, while walking prograde at $1 \mathrm{~m} / \mathrm{s}$, radius $12.2 \mathrm{~m}$, tangential velocity $6.0 \mathrm{~m} / \mathrm{s}$ (Table 1 row 2). 


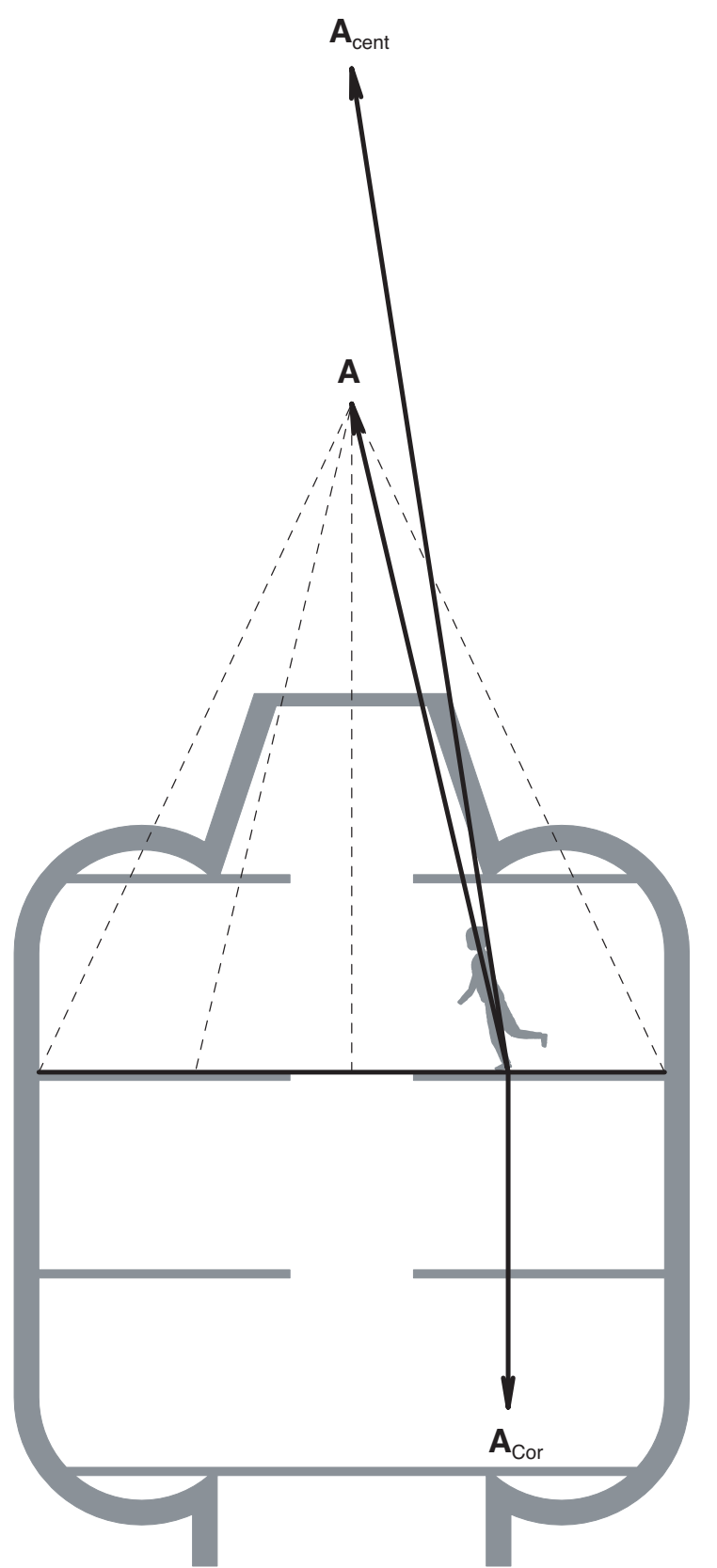

Figure 6: Centripetal, Coriolis, and total acceleration while walking retrograde at $1 \mathrm{~m} / \mathrm{s}$, radius $12.2 \mathrm{~m}$, tangential velocity $6.0 \mathrm{~m} / \mathrm{s}$ (Table 1 row 2).

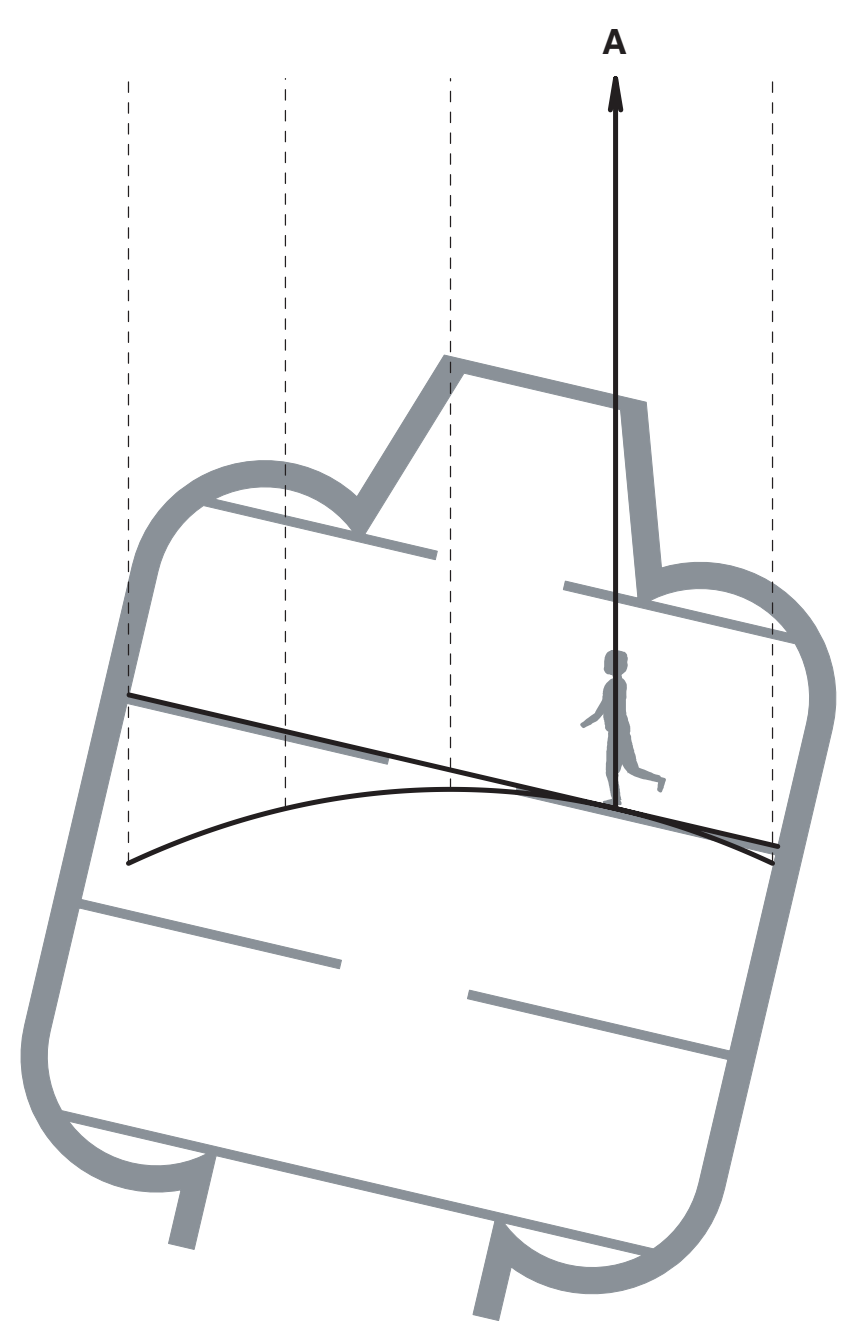

Figure 7: Apparent gravity for the inhabitant in Figure 6, while walking retrograde at $1 \mathrm{~m} / \mathrm{s}$, radius $12.2 \mathrm{~m}$, tangential velocity $6.0 \mathrm{~m} / \mathrm{s}$ (Table 1 row 2). 


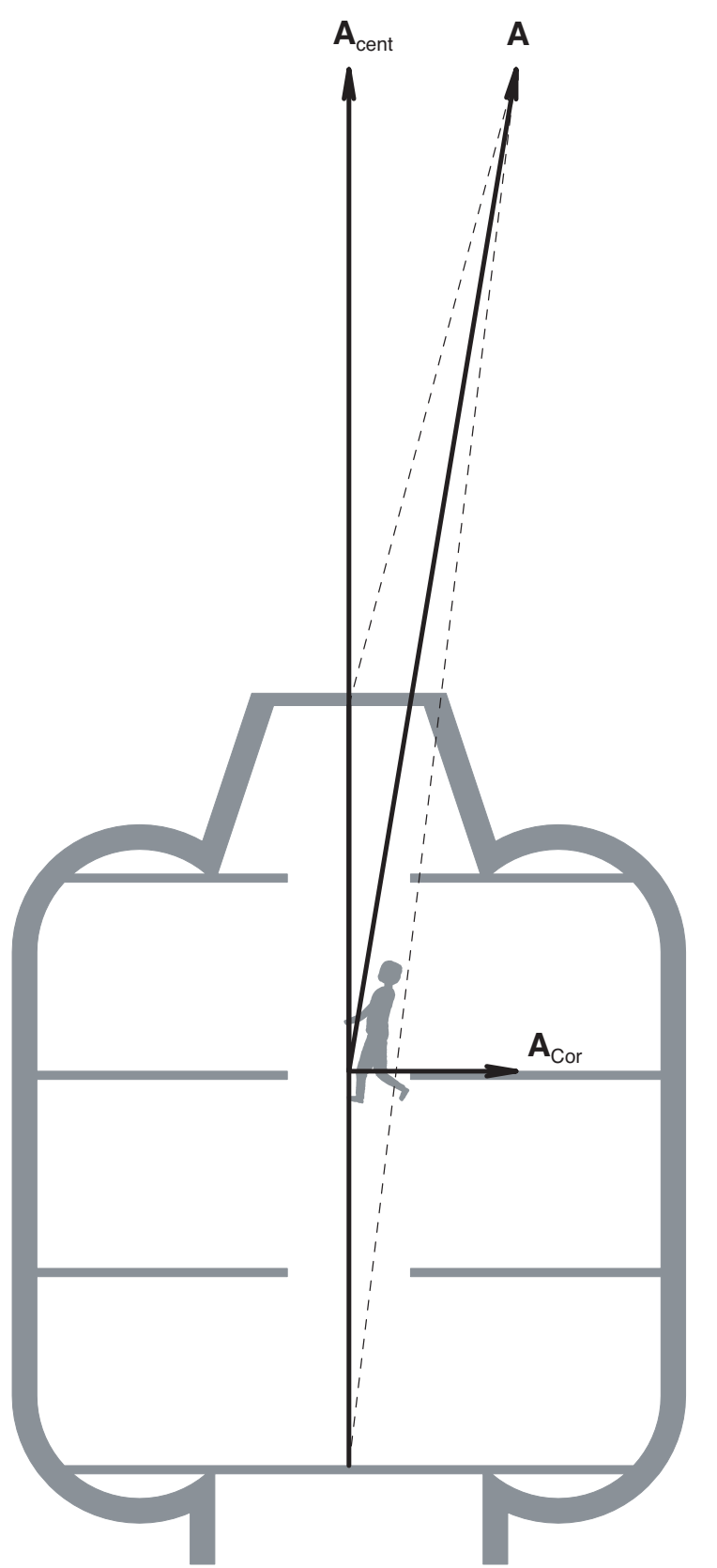

Figure 8: Centripetal, Coriolis, and total acceleration while descending at $0.5 \mathrm{~m} / \mathrm{s}$, radius $12.2 \mathrm{~m}$, tangential velocity $6.0 \mathrm{~m} / \mathrm{s}$ (Table 1 row 2 ).

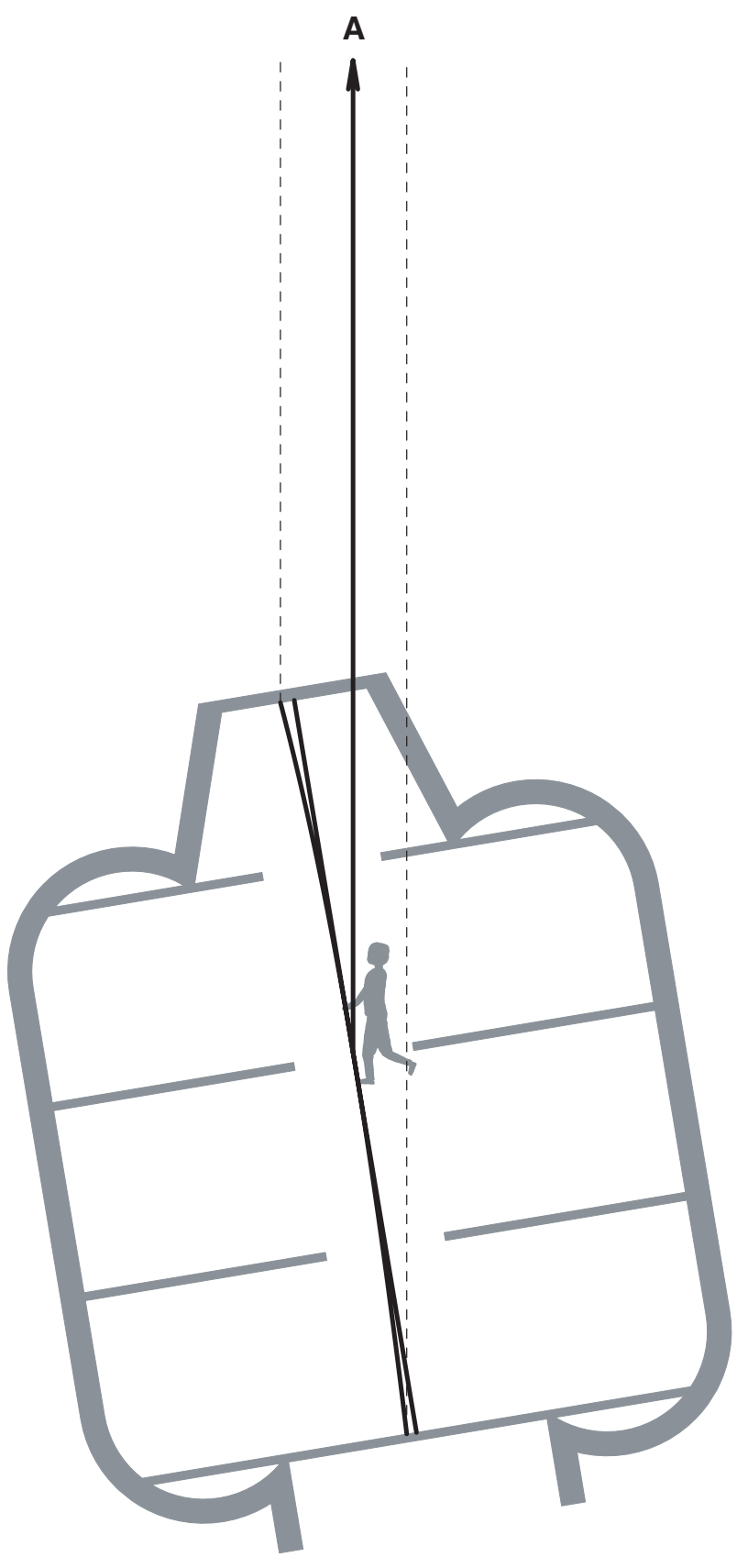

Figure 9: Apparent gravity for the inhabitant in Figure 8, while descending at $0.5 \mathrm{~m} / \mathrm{s}$, radius $12.2 \mathrm{~m}$, tangential velocity $6.0 \mathrm{~m} / \mathrm{s}$ (Table 1 row 2). 


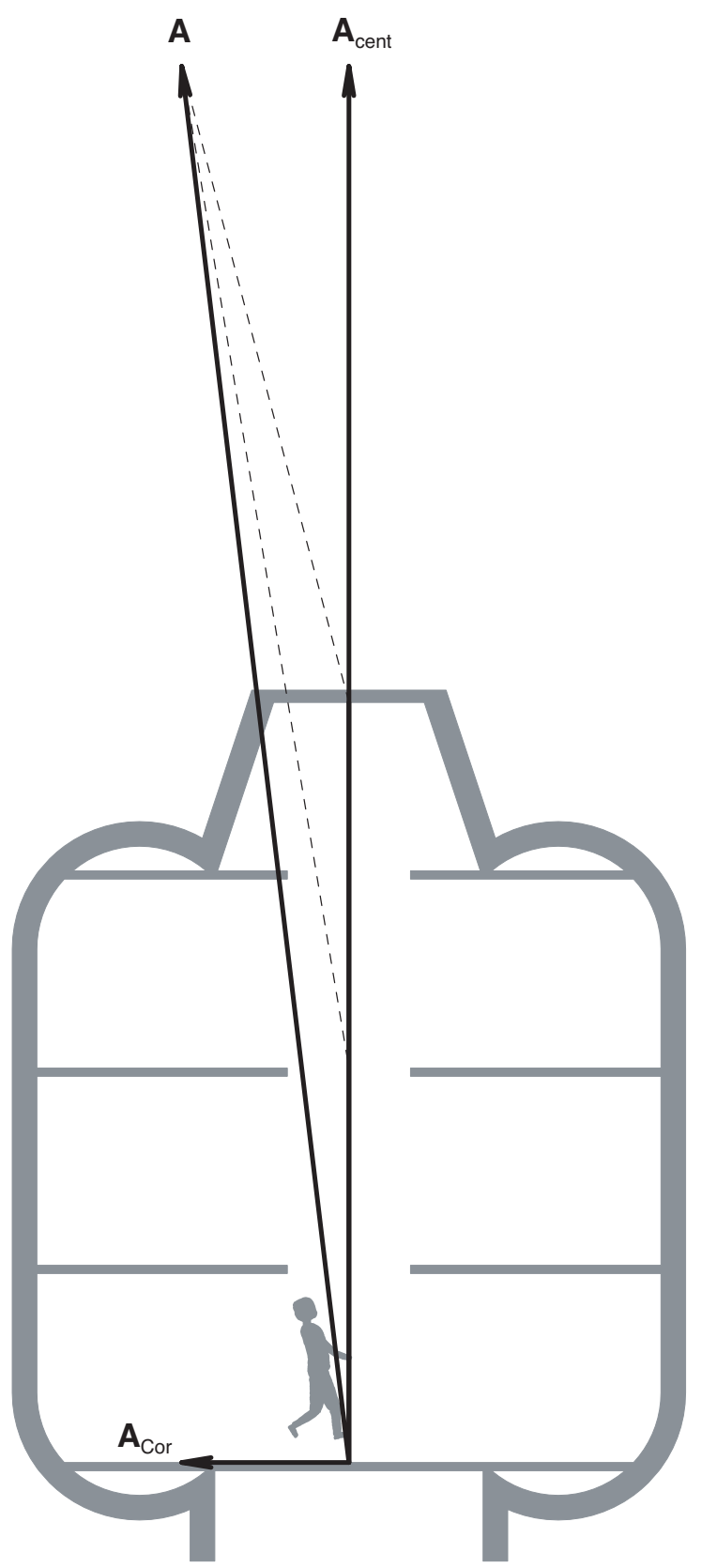

Figure 10: Centripetal, Coriolis, and total acceleration while ascending at $0.5 \mathrm{~m} / \mathrm{s}$, radius $17.0 \mathrm{~m}(12.2+4.8)$, tangential velocity $8.3 \mathrm{~m} / \mathrm{s}$.

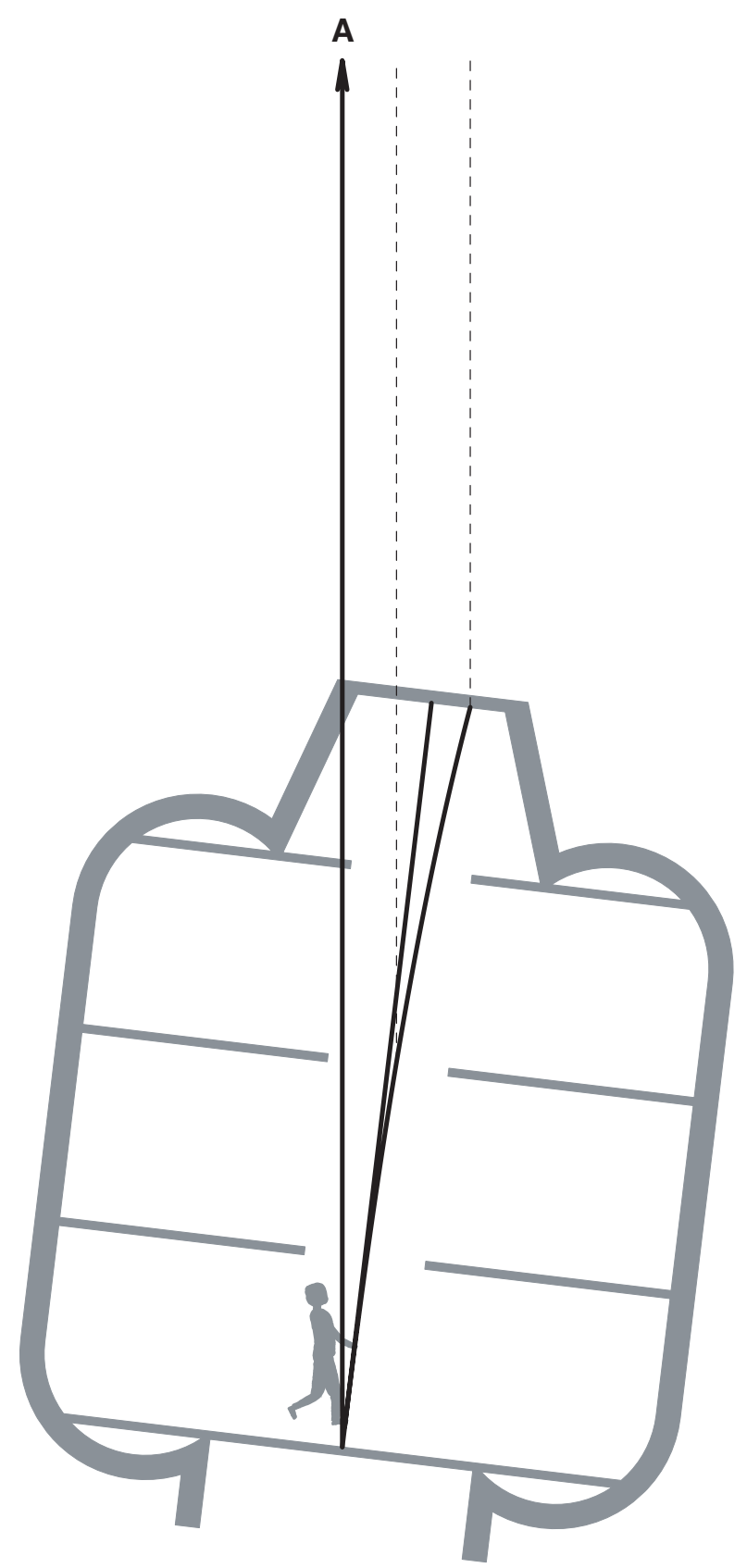

Figure 11: Apparent gravity for the inhabitant in Figure 10, while ascending at $0.5 \mathrm{~m} / \mathrm{s}$, radius $17.0 \mathrm{~m}(12.2+4.8)$, tangential velocity $8.3 \mathrm{~m} / \mathrm{s}$. 


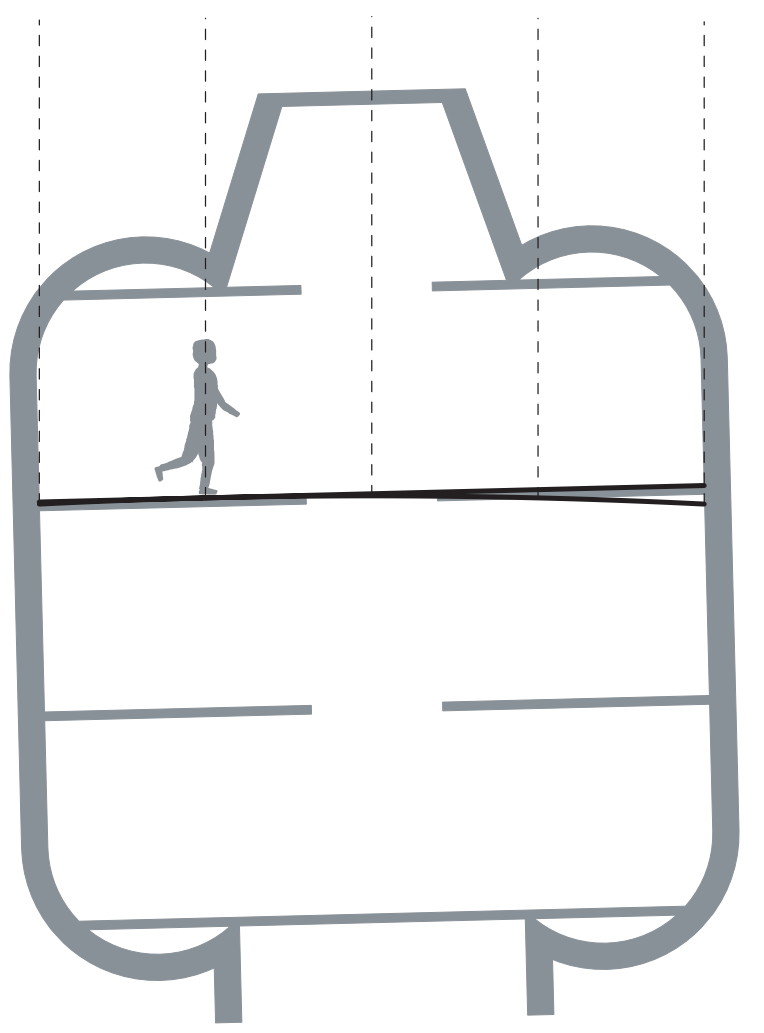

Figure 12: Apparent gravity while walking prograde at $1 \mathrm{~m} / \mathrm{s}$, radius $67.1 \mathrm{~m}$, tangential velocity $14.0 \mathrm{~m} / \mathrm{s}$ (Table 1 row 3 ). Compare Figure 5.

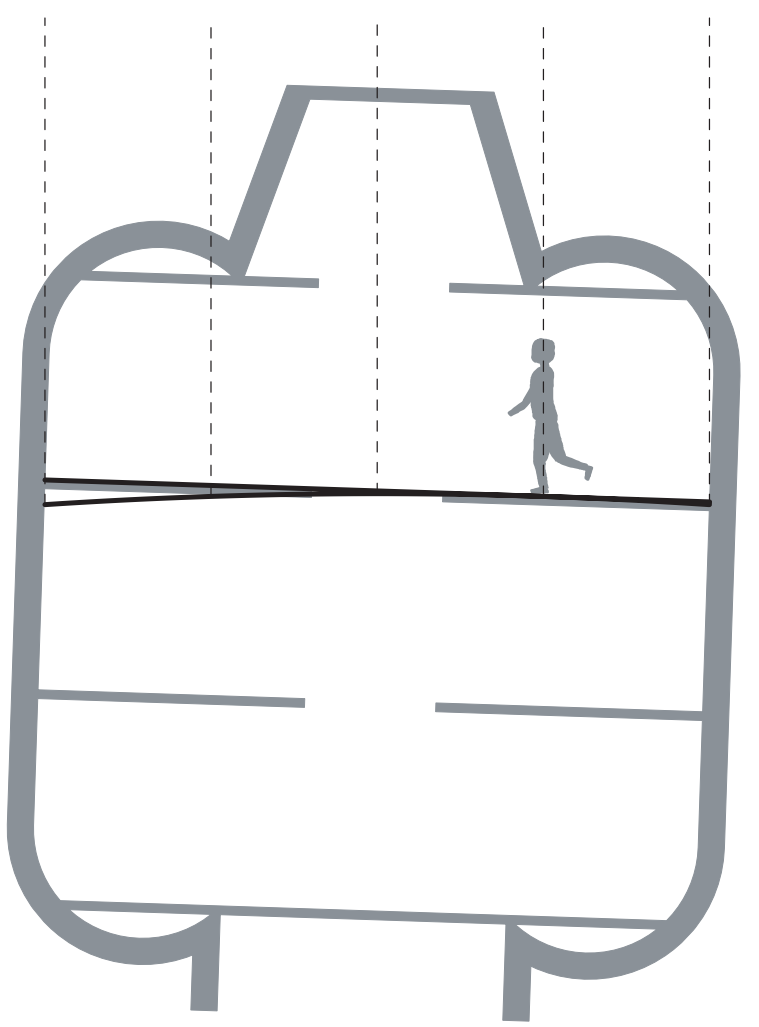

Figure 13: Apparent gravity while walking retrograde at $1 \mathrm{~m} / \mathrm{s}$, radius $67.1 \mathrm{~m}$, tangential velocity $14.0 \mathrm{~m} / \mathrm{s}$ (Table 1 row 3 ). Compare Figure 7.

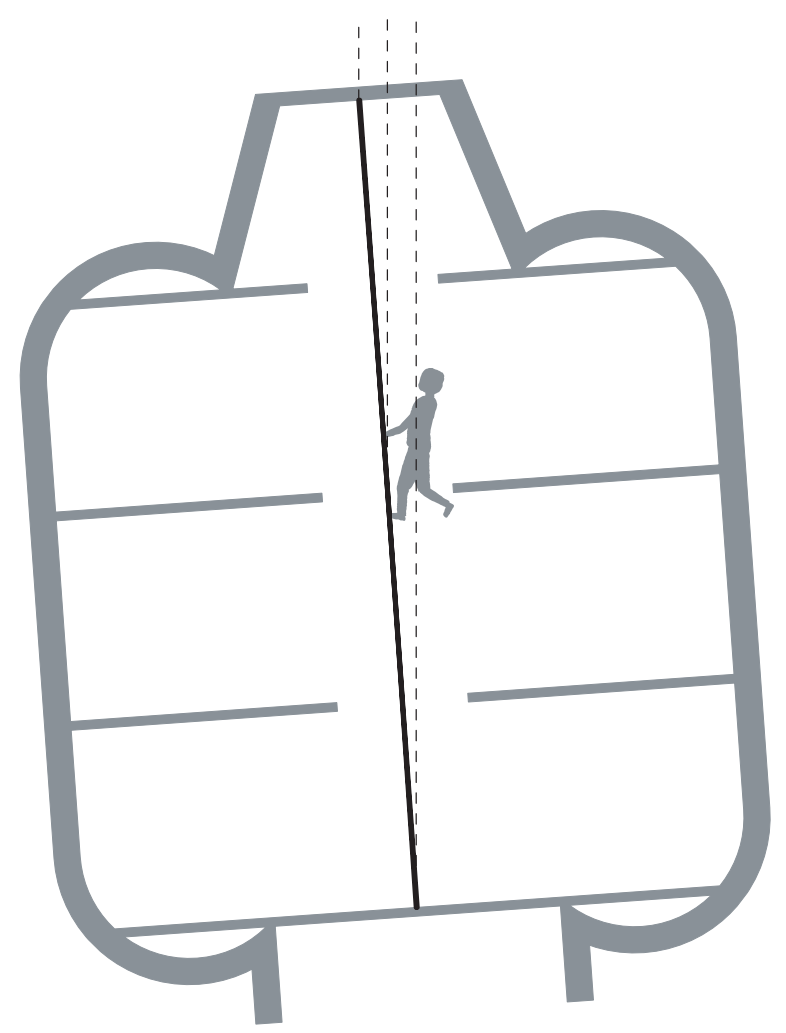

Figure 14: Apparent gravity while descending at $0.5 \mathrm{~m} / \mathrm{s}$, radius 67.1 $\mathrm{m}$, tangential velocity $14.0 \mathrm{~m} / \mathrm{s}$ (Table 1 row 3 ). Compare Figure 9.

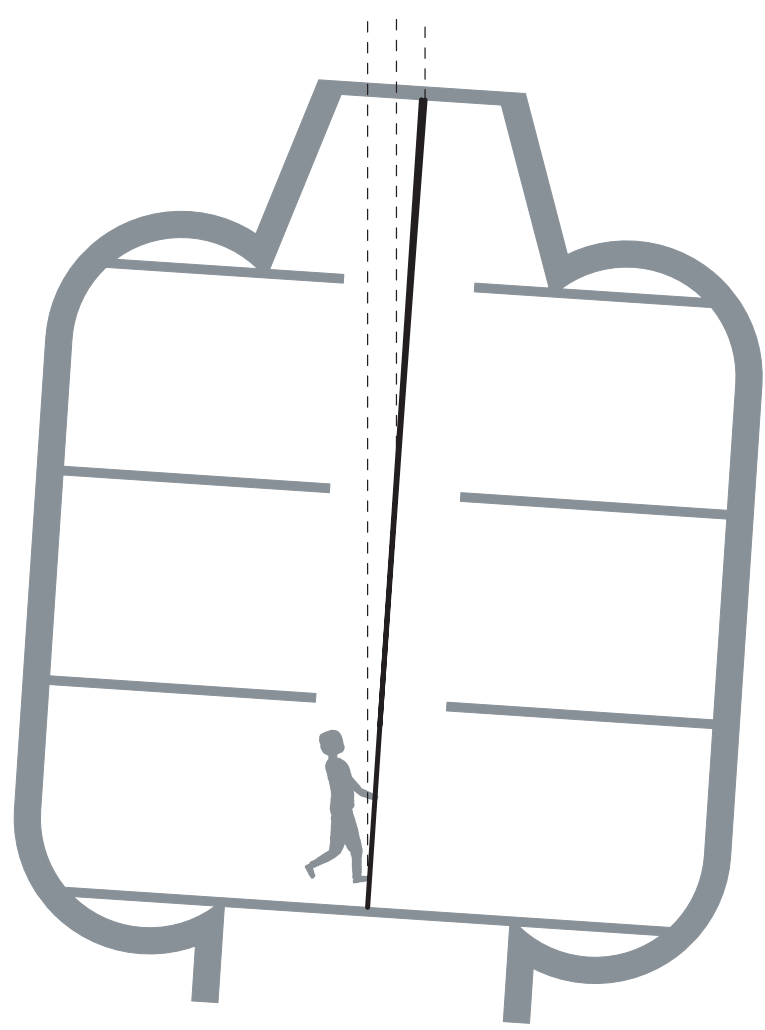

Figure 15: Apparent gravity while ascending at $0.5 \mathrm{~m} / \mathrm{s}$, radius $71.9 \mathrm{~m}$ $(67.1+4.8)$, tangential velocity $15.1 \mathrm{~m} / \mathrm{s}$. Compare Figure 11 . 


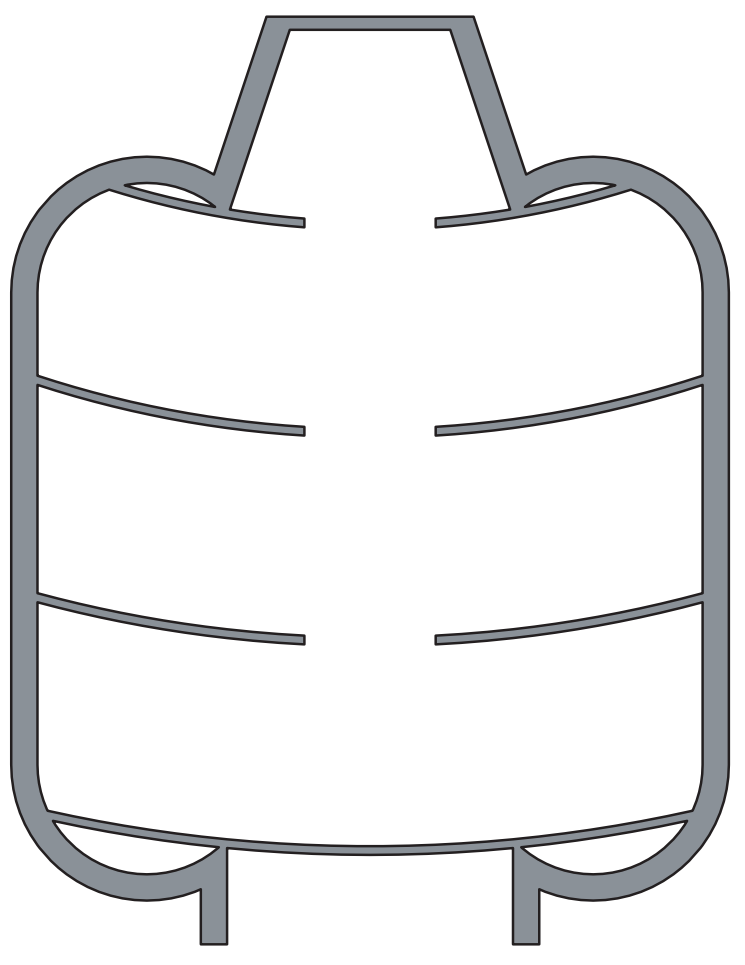

Figure 16: Floor curvature for a top-floor radius of 12.2 meters (Table 1 row 2).

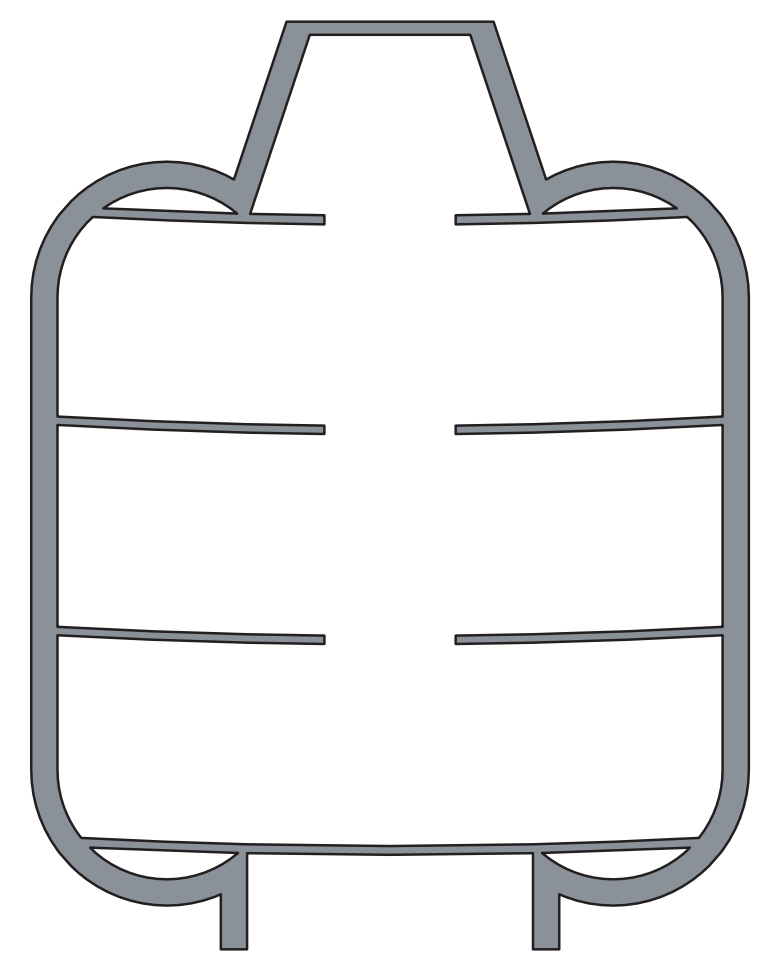

Figure 17: Floor curvature for a top-floor radius of 67.1 meters (Table 1 row 3 ). the bounds of "comfortable" ramps. The ladder slope varies by less than one degree from top to bottom, which for reasons of safety as well as comfort is probably a good thing.

The apparent floor slope, in the inhabitant's frame of reference, can be avoided entirely if the floor is curved, as shown in Figures 16 and 17. Because the Coriolis acceleration is always perpendicular to the relative velocity, a circular floor aligns the Coriolis and centripetal accelerations and remains perpendicular to both. Figure 16 shows that the required curvature at the extreme minimum radius is substantial; Figure 17 shows that a larger radius is not difficult to accommodate.

\section{LESSONS FOR MINIMAL ARTIFICIAL-GRAVITY HABITATS}

MODULE ORIENTATION - For structural integrity as a pressure vessel as well as for ease of manufacture and launch, a minimal space habitat is likely to be composed of right circular cylinders. If motion within a minimal habitat is principally parallel to the cylinder's geometric axis, with little room to move in the cross section, then the preceding analysis offers some lessons for the best orientation of such a cylinder with respect to the rotation axis for artificial gravity. These lessons also apply to the interior planning of larger habitats in which internal motion in several directions is possible but not equally desirable.

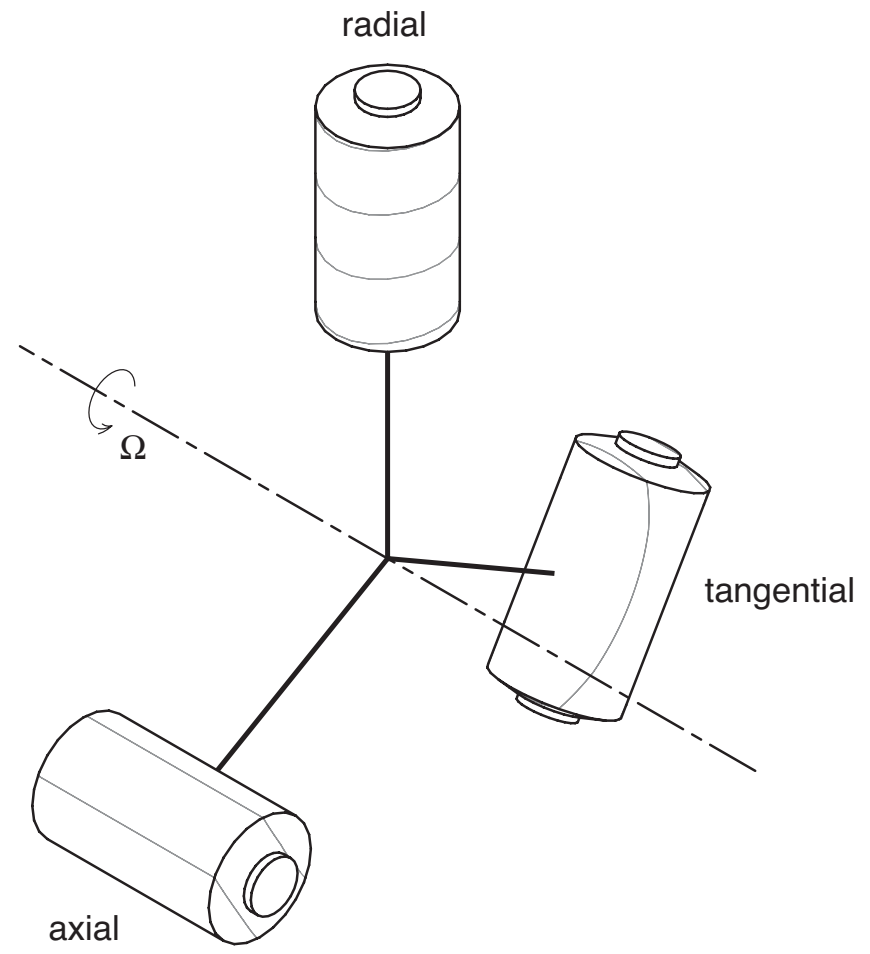

Figure 18: Three principal orientations of a cylindrical habitat with respect to the axis of rotation. 
Figure 18 illustrates the three principal orientations of a smaller cylindrical habitat with respect to the rotation axis. (For the sake of illustration, it uses the dimensions of the ISS "Destiny" laboratory module.) The following paragraphs discuss the relative merits of each orientation for artificial gravity.

Axial Orientation - Considering only the gravity environment within a minimal habitat, ideally, the geometric axis of the cylinder would be parallel to the axis of rotation. This is because motion parallel to that axis involves no Coriolis accelerations, floor curvature, or apparent slope. Furthermore, if furniture, equipment, controls and displays are arranged along the sides of such a cylinder, then users will tend to face prograde or retrograde. In this orientation, head tilt (up and down on its pitch axis) is also parallel to the habitat's rotation, and incurs no cross-coupling, rotational illusions, or dizziness. (Alas, head yaw is always perpendicular to, and cross-couples with, the habitat's rotation.)

Unfortunately, from the standpoint of spacecraft dynamics, this may be the least stable orientation. Schultz, Rupp, Hajos, and Butler [1989] applied an endbody dynamic analysis to cylindrical habitat modules suspended from a tether, and found that a small misalignment with the rotation axis (caused by twisting of the tether) would tend to grow rather than dampen. Based on this analysis, they recommended a 90-degree realignment, placing the cylinder axis tangent to the rotation.

Few spacecraft concepts have adopted the axial orientation, perhaps because of its inherent instability. Figure 19 is one notable example, developed in considerable detail by engineers at the Lockheed Aircraft Corporation in 1960 [Kramer \& Byers, 1960]. Their concept relied on a rigid structure and a substantial stabilization system to maintain the configuration.

Tangential Orientation - This is more stable than the axial orientation. Of course, stability is a good thing for promoting comfort. However, the tangential orientation involves either apparent floor slope or actual floor curvature. The necessary curvature is not difficult to accommodate as long as the module length is small in proportion to the rotational radius. However, this tends to impose more limits on the comfort zone for artificial gravity: either a "short" module, or a "large" rotational radius. The latter option increases the kinetic energy for any particular habitat mass and level of artificial gravity.

Most artificial-gravity concepts adopt this orientation. In particular, toroids are rings of tangential modules. A prime example of a minimal habitat in this orientation is the "straw man" design for a variable gravity research facility (VGRF) described by Lemke [1988]. Figure 20 shows the concept. Though it's not obvious in the

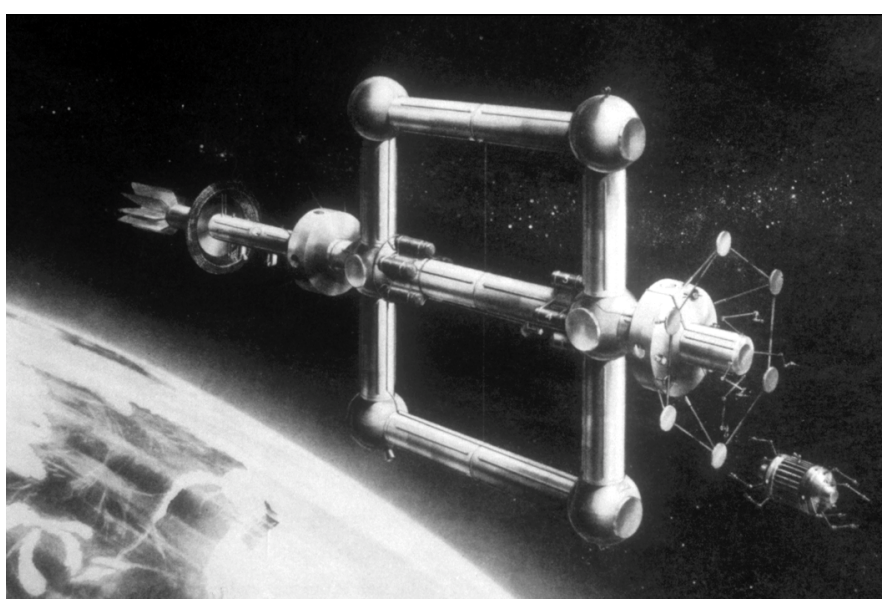

Figure 19: Artificial-gravity space station, axial orientation [Kramer \& Byers, 1960].

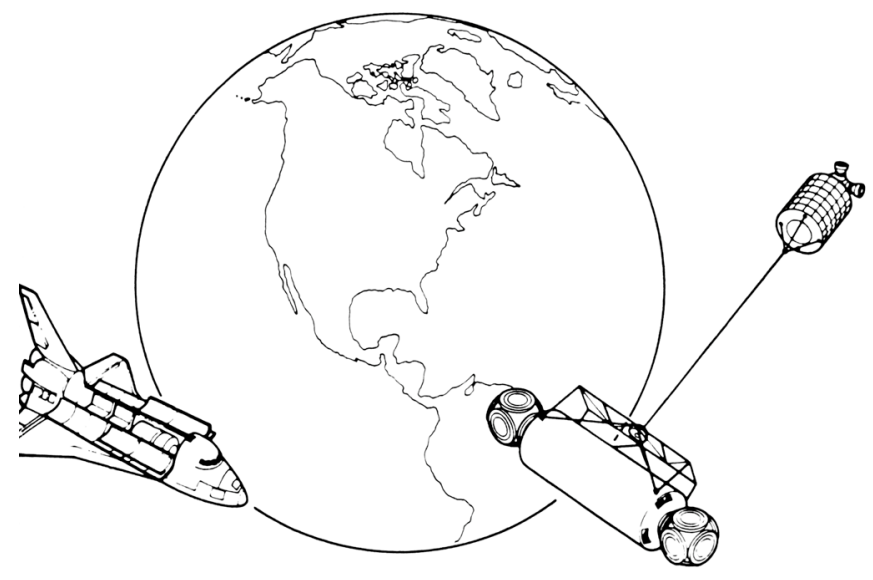

Figure 20: Variable gravity research facility, tangential orientation [Lemke, 1988].

drawing, the specified orientation of the habitat module is tangential (not axial). With the tether fully extended, the rotational radius to the module floor would be much greater than the module length: about 228 meters versus 9 meters. Therefore, the apparent floor slope would be quite small.

Radial Orientation - This may be the least comfortable orientation of all. It forces movement between decks with different apparent gravity levels. If the change in gravity is too large or too sudden, the resulting shift of bodily fluids may lead to fainting - especially when descending from a weaker gravity level to a stronger one. The apparent slope of a ladder depends on whether one is ascending or descending, and one needs to take care to use the side of the ladder appropriate to one's direction of motion. Climbing is generally slower than 


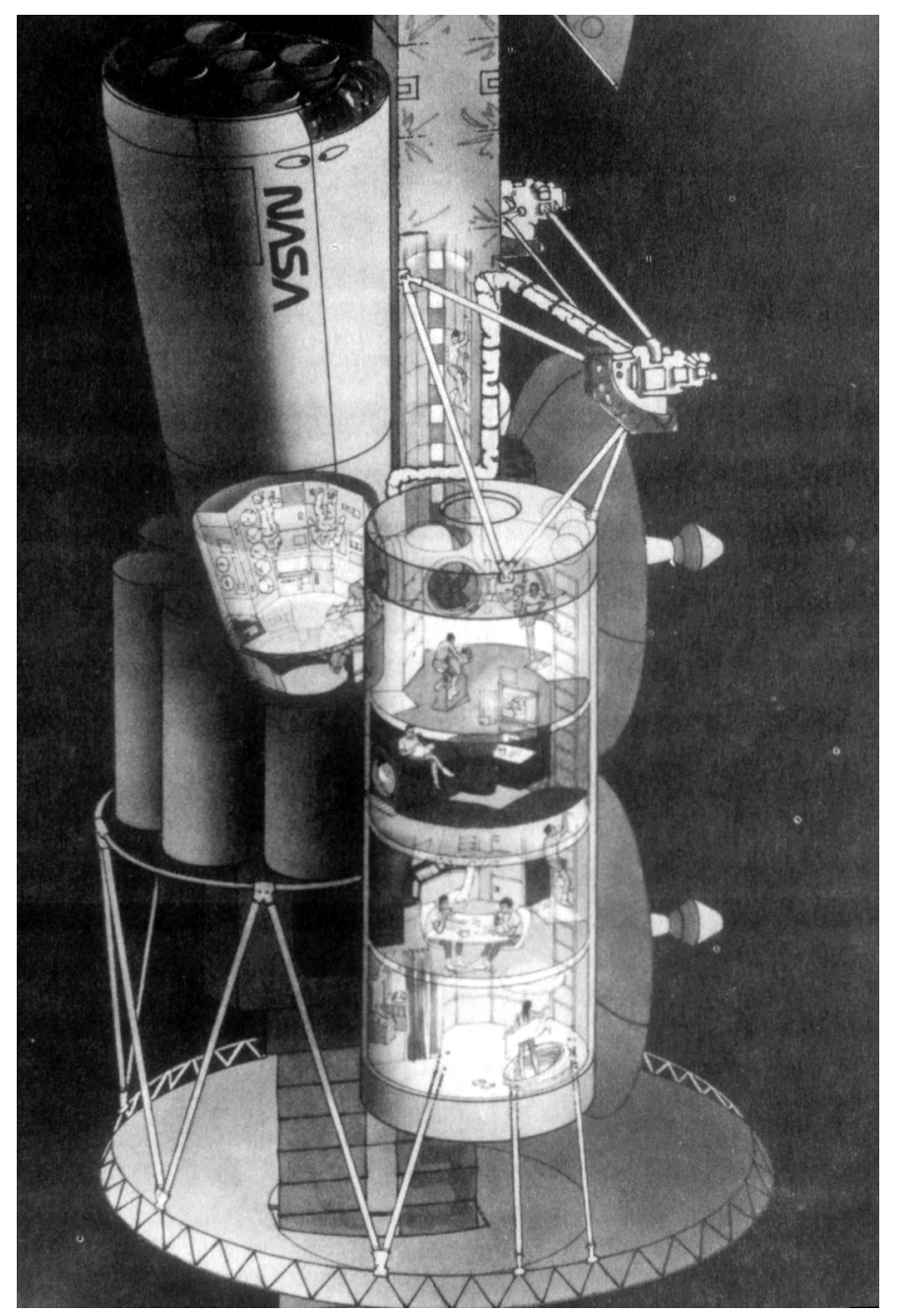

Figure 21: Variable gravity research station, radial orientation [Staehle, 1989]. Illustration by Carter Emmart.

walking, and occupies the hands as well as the feet, making it difficult to carry anything. Finally, this orientation leads to a circular plan, with an arrangement of furniture and equipment that's indifferent to the plane of rotation, ignores the apparent gravitational distinction between prograde and retrograde, and promotes head yaw that cross-couples with the habitat rotation.

Nevertheless, there are a few spacecraft concepts that have adopted this orientation. Figure 21 shows the variable gravity research station proposed by Staehle [1989], based in turn on the interplanetary habitat described by Welch [1985]. A close look at the drawing reveals a ladder mounted to the module wall on the righthand side, behind the communications dishes. Assuming the dishes have a fixed orientation, in the plane of rotation, this places the ladder in the rotation plane as well. In this orientation, due to Coriolis accelerations, the ladder will seem to lean sideways, making it quite uncomfortable to climb. It should be turned perpendicular to the plane of rotation.
PSYCHOLOGICAL ORIENTATION - Aside from the physical orientation of the habitat modules, one must also consider the psychological orientation of the inhabitants. The gravitational distortions due to Coriolis accelerations and cross-coupled rotations occur only during motion within the rotating environment, in proportion to the relative velocity. A person awakening in bed in the morning or engaged in some sedentary activity might forget about these effects, or lose track of their orientation with respect to the environment, only to be rudely reminded when he rises from his bed or chair or turns to his side. Anything that helps a person to maintain his bearings with respect to the direction of rotation would allow him to prepare himself for the consequences of his actions, aiding his coordination and adaptation to the rotating environment.

Hesselgren [1967, 1975] builds his theory of architecture on the foundations of perception psychology. He expounds on the importance of gestalts in composing comprehensible environments, and describes "transformation tendencies" wherein a perception in one modality (e.g., visual texture) may produce a mental image or expectation of a perception in another modality (e.g., tactile grain).

One modality that he does not discuss - that is taken for granted on Earth but cannot be in space - is vestibular perception. It may be possible, through experience in a properly designed environment, to acquire a transformation tendency to vestibular perception from visual, acoustic, haptic, or other perceptions. The goal is to provide reminders that motion relative to these cues will result in certain inescapable side effects, inherent in the artificial gravity. For example, just as ceilings are often lighter in color than floors, one might distinguish the prograde (east) and retrograde (west) walls with advancing and receding colors or bas-relief shapes. Such cues might act as signals, triggering adaptive coordination in the inhabitants. From the designer's point of view, a consistent "vocabulary" of such signals would have to arise from convention. From the inhabitant's point of view, these conventions might to some extent be taught, but the spontaneous transformation to a vestibular image would rely on association based on direct experience.

\section{ALTERNATIVES TO ROTATING THE HABITAT}

ON-BOARD SHORT-ARM CENTRIFUGE - The limits in Table 1 derive from experiments in rotating rooms and space station simulators that required subjects to maintain a certain level of activity, moving about within the rotating environment. More recent studies have focused on short-arm centrifuges and rotating beds, wherein subjects are required to do little other than lay still and relax [Cardús, Diamandis, McTaggart, \& Campbell, 1990; Cardús, McTaggart, \& Campbell, 1991; Cardús \& McTaggart, 1993]. This latter strategy appears to permit a much smaller radius and greater angular 
velocity than suggested by Table 1, raising the hope that an on-board centrifuge may be a viable alternative to rotating the entire habitat.

Several questions regarding the efficacy of this approach come to mind at the outset. While Earth-based experiments may offer some indications, ultimately these must be studied in orbit:

- What is the trade-off between intensity and duration? Is the therapeutic gravity dose a simple integral of intensity with elapsed time?

- If inhabitants must remain motionless to avoid motion sickness during high-speed centrifugation, can they engage in any significant activity, either work-related, recreational, or therapeutic?

- If the required daily exposure requires several hours of non-activity, is it best scheduled during the sleep period? If so, can inhabitants sleep satisfactorily while upright in an artificial-gravity environment, after spending their waking hours in weightlessness?

The mechanical design also entails some problems. The centrifuge must couple to some counter-rotating mass - possibly another centrifuge. If the habitat itself is allowed to counter-rotate, it will be pervaded by some level of artificial gravity that's likely to be rather uncomfortable for any sort of activity due to the short radius. (Comfort in the short-arm centrifuge depends on inactivity.) The centrifuge must also balance across its spin axis to avoid imparting a wobble to the habitat.

One possible design would be to arrange users in groups of four, on two parallel counter-rotating disks, with two people balanced across the diameter of each disk. This may be problematic for treatments of more than a few hours, especially in the case of a rotating bed. If any one of the four wants to leave the centrifuge (for example, to use the toilet) he or she must first stop the rotation of the entire mechanism, interrupting the treatment - or even the sleep - of the other three users as well. If an independent centrifuge is allocated to each person, then sufficient mass must be identified and allocated for the balance and counter-spin. This will also require more volume, since each centrifuge requires its own disk of space to rotate through. A single centrifuge might be shared by users in different shifts, if the treatment period is short enough. However, dividing a small crew into distinctly different shifts, planned around each individual's hours of isolation in the centrifuge, may disrupt the crew's social cohesiveness.

EXERCISE, DIET AND MEDICATION - These have been ineffective at preventing bone demineralization during exposure to micro gravity. While terrestrial medical science is advancing rapidly, and an effective medicinal countermeasure might yet be found, it must be noted that micro gravity itself has a significant influence on the body's response to medication, due to fluid shift, fluid loss, and cell membrane thickening. Furthermore, gravity is an important factor in directing the development of bone tissue where it's needed, at stress points [Chaffin \& Andersson, 1984, p. 25; Connors et al., 1985, p. 20; Marwick, 1986, p. 2020; Merz, 1986, p. 2043; Mohler, 1962; Oberg \& Oberg, 1986, p. 131-132; Woodard \& Oberg, 1984, p. 175]. A treatment that prompts bone tissue development in the absence of gravity may act indiscriminately, bringing no real benefit and perhaps even harm.

\section{CONCLUSION}

A minimalist approach to the design of an artificial-gravity environment will undoubtedly require the inhabitants to adapt, over a period of time, to unfamiliar forces and torques. Micro gravity also requires a short period of adaptation, with discomfort and inconvenience, followed by continual physiological deconditioning. Therefore, even if artificial gravity requires an equal period of adaptation, it's still preferable to micro gravity for health preservation.

To ease the inhabitants' adaptation to artificial-gravity, the habitat designer must be aware of the forces and torques that will impinge them in their routine activities, and arrange things as much as possible to their advantage. It's not acceptable to mindlessly adopt plans and elements originally designed for either terrestrial gravity or micro gravity; artificial gravity demands its own paradigm. In particular, motion in the "horizontal" plane (perpendicular to the radius of rotation) is not isotropic: the prograde (east), retrograde (west), and axial (northsouth) directions emerge as gravitationally distinct, as exhibited by the relative motion of free-falling particles, the orientation of Coriolis accelerations, and the apparent slope of planar surfaces.

Inhabitants are likely to adapt easiest if they can maintain their mental orientation with respect to the habitat rotation, allowing them to adjust their exertions in anticipation of the gravitational distortions. Aside from the layout of partitions, furniture, and equipment, other visual cues, such as color or form, might support this.

Artificial gravity adds considerable complexity to space habitat design. However, it is not clear that alternatives such as on-board centrifuges, medical treatments, or post-flight rehabilitation are any less complex especially if the destination is other than Earth.

\section{REFERENCES}

1. BOCA (1989). The BOCA National Building Code, 1990 (11th edition). Country Club Hills, Illinois, USA: Building Officials and Code Administrators, Inc.

2. Capps, Stephen; Fowler, Robert; Appleby, Matthew (1991). "Induced Gravity Mars Transportation Systems: Configuration and Hardware Penalties." Space 
Manufacturing 8 - Energy and Materials from Space: Proceedings of the Tenth Princeton / AIAA / SSI Conference, May 15-18, 1991, p. 126-131. Reston, Virginia, USA: American Institute of Aeronautics and Astronautics.

3. Cardús, David; Diamandis, Peter; McTaggart, Wesley G.; Campbell, Scott (1990). "Development of an Artificial Gravity Sleeper (AGS)." The Physiologist, vol. 33, no. 1, supplement, p. S112-S113. Bethesda, Maryland, USA: American Physiological Society.

4. Cardús, David; McTaggart, Wesley G.; Campbell, Scott (1991). "Progress in the Development of an Artificial Gravity Simulator (AGS)." The Physiologist, vol. 34, no. 1, supplement, p. S224-S225. Bethesda, Maryland, USA: American Physiological Society.

5. Cardús, David; McTaggart, Wesley G. (1993). "The Cardiovascular Response to the AGS." The Physiologist, vol. 36, no. 1, supplement, p. S155-S157. Bethesda, Maryland, USA: American Physiological Society.

6. Chaffin, Don B.; Andersson, Gunnar B. J. (1984). Occupational Biomechanics. New York, New York, USA: John Wiley and Sons.

7. Clark, Carl C.; Hardy, James D. (1960). "Gravity Problems in Manned Space Stations." Proceedings of the Manned Space Stations Symposium, April 20-22, 1960, p. 104-113. New York, New York, USA: Institute of the Aeronautical Sciences.

8. Connors, Mary M.; Harrison, Albert A.; Akins, Faren R. (1985). Living Aloft: Human Requirements for Extended Spaceflight, NASA SP 483. Washington, DC, USA: National Aeronautics and Space Administration.

9. Cramer, D. Bryant (1985). "Physiological Considerations of Artificial Gravity." Applications of Tethers in Space, NASA CP 2364, vol. 1, p. 3.95-3.107. Washington, DC, USA: National Aeronautics and Space Administration.

10. Gilruth, Robert R. (1969). "Manned Space Stations Gateway to Our Future in Space." Manned Laboratories in Space, p. 1-10. New York, New York, USA: SpringerVerlag.

11. Gordon, Theodore J.; Gervais, Robert L. (1969). "Critical Engineering Problems of Space Stations." Manned Laboratories in Space, p. 11-32. New York, New York, USA: Springer-Verlag.

12. Graybiel, Ashton (1977). "Some Physiological Effects of Alternation Between Zero Gravity and One Gravity." Space Manufacturing Facilities (Space Colonies): Proceedings of the Princeton / AIAA / NASA Conference, May 7-9, 1975, p. 137-149. Reston, Virginia, USA: American Institute of Aeronautics and Astronautics.

13. Hall, Theodore W. (1999). Inhabiting Artificial Gravity, AIAA 99-4524. AIAA Space Technology Conference, Albuquerque, New Mexico, USA, 28-30 September 1999. Reston, Virginia, USA: American Institute of Aeronautics and Astronautics.

14. Hesselgren, Sven (1967). The Language of Architecture. Lund, Sweden: Studentlitteratur.

15. Hesselgren, Sven (1975). Man's Perception of Man-Made Environment: An Architectural Theory. Lund, Sweden: Studentlitteratur.
16. Hill, Paul R.; Schnitzer, Emanuel (1962 September). "Rotating Manned Space Stations." Astronautics, vol. 7, no. 9, p. 14-18. New York, New York, USA: American Rocket Society.

17. Kennedy, Kriss J. (1999). ISS TransHab: Architecture Description, SAE 1999-01-2143. 29th International Conference on Environmental Systems (ICES), Denver, Colorado, USA, 12-15 July 1999. Warrendale, Pennsylvania, USA: Society of Automotive Engineers.

18. Kramer, Saunders B.; Byers, Richard A. (1960). "A Modular Concept for a Multi-Manned Space Station." Proceedings of the Manned Space Stations Symposium, April 20-22, 1960, p. 36-72. New York, New York, USA: Institute of the Aeronautical Sciences.

19. Lally, Eugene F. (1962 September). "To Spin or Not To Spin." Astronautics, vol. 7, no. 9, p. 56-58. New York, New York, USA: American Rocket Society.

20. Lemke, L. G. (1988). "VGRF Technology Overview and Strawman Design." NASA Ames Research Center, 27 March 1988.

21. Marwick, Charles (1986 October 17). "Physicians Called Upon to Help Chart Future Space Effort." Journal of the American Medical Association, vol. 256, no. 15, p. 2015+. Chicago, Illinois, USA: American Medical Association.

22. Merz, Beverly (1986 October 17). "The Body Pays a Penalty for Defying the Law of Gravity." Journal of the American Medical Association, vol. 256, no. 15, p. 2040+. Chicago, Illinois, USA: American Medical Association.

23. Mohler, Stanley R. (1962 May). "Aging and Space Travel." Aerospace Medicine, vol. 33, no. 5, p. 594-597. Alexandria, Virginia, USA: Aerospace Medical Association.

24. Oberg, James E.; Oberg, Alcestis R. (1986). Pioneering Space: Living on the Next Frontier. New York, New York, USA: McGraw-Hill.

25. Raymond, Chris Anne (1986 October 17). "Physicians Trade White Coats for Space Suits." Journal of the American Medical Association, vol. 256, no. 15, p. 2033+. Chicago, Illinois, USA: American Medical Association.

26. Schultz, David N.; Rupp, Charles C.; Hajos, Gregory A.; Butler, John M. (1989). A Manned Mars Artificial Gravity Vehicle, AAS 87-203. The Case for Mars III: Strategies for Exploration - General Interest and Overview, p. 325-352. Vol. 74, Science and Technology Series, American Astronautical Society. San Diego, California, USA: Univelt, Inc.

27. Staehle, Robert L. (1989). Earth Orbital Preparations for Mars Expeditions, AAS 87-205. The Case for Mars III: Strategies for Exploration - General Interest and Overview, p. 373-396. Vol. 74, Science and Technology Series, American Astronautical Society. San Diego, California, USA: Univelt, Inc.

28. Stone, Ralph W. (1973). "An Overview of Artificial Gravity." Fifth Symposium on the Role of the Vestibular Organs in Space Exploration, NASA SP 115, p. 23-33. Washington, DC, USA: National Aeronautics and Space Administration.

29. Welch, Steven (1985). Mission Strategy and Spacecraft Design for a Mars Base Program, AAS 84-169. The Case for Mars II, p. 345-375. Vol. 62, Science and Technology 
Series, American Astronautical Society. San Diego, California, USA: Univelt, Inc.

30. Woodard, Daniel; Oberg, Alcestis R. (1984). The Medical Aspects of a Flight to Mars, AAS 81-239. The Case For Mars, p. 173-180. Vol. 57, Science and Technology Series, American Astronautical Society. San Diego, California, USA: Univelt, Inc.

31. Zubrin, Robert M.; Baker, David A.; Gwynne, Owen (1991). Mars Direct: A Simple, Robust, and Cost Effective Architecture for the Space Exploration Initiative, AIAA-910328. Reston, Virginia, USA: American Institute of Aeronautics and Astronautics.

\section{CONTACT}

Theodore W. Hall completed his doctoral degree in architecture at the University of Michigan in 1994. He is currently a research officer in the Department of Architecture at the Chinese University of Hong Kong, engaged in software development for computer-aided design, scientific visualization, and virtual reality.

Postal address: Theodore W. Hall; Department of Architecture; Chinese University of Hong Kong; Sha Tin, Hong Kong; China.

e-mail: twhall@cuhk.edu.hk

\section{ADDITIONAL SOURCES}

http://www0.arch.cuhk.edu.hk/ hall/ag/ 